\title{
Extraordinary Control of Terahertz Beam Reflectance in Graphene Electro-absorption Modulators
}

\author{
Berardi Sensale-Rodriguez, ${ }^{* \dagger}$ Rusen Yan, ${ }^{\dagger}{ }^{\ddagger}$ Subrina Rafique, ${ }^{\dagger}$ Mingda Zhu, ${ }^{\dagger}$ Wei Li, ${ }^{\ddagger}, \S$ Xuelei Liang, ${ }^{\S}$ \\ David Gundlach, ${ }^{\ddagger}$ Vladimir Protasenko, ${ }^{\dagger}$ Michelle M. Kelly, ${ }^{\dagger}$ Debdeep Jena, ${ }^{\dagger}$ Lei Liu, ${ }^{\dagger}$ \\ and Huili Grace Xing*,†
}

\begin{abstract}
${ }^{\dagger}$ Department of Electrical Engineering, University of Notre Dame, Notre Dame, Indiana 46556, United States
${ }^{\ddagger}$ Semiconductor and Dimensional Metrology Division, National Institute of Standards and Technology, Gaithersburg, Maryland 20899, United States

${ }^{\S}$ Key Laboratory for the Physics and Chemistry of Nanodevices and Department of Electronics, Peking University, Beijing, 100871, China
\end{abstract}

ABSTRACT: We demonstrate a graphene-based electroabsorption modulator achieving extraordinary control of terahertz reflectance. By concentrating the electric field intensity in an active layer of graphene, an extraordinary modulation depth of $64 \%$ is achieved while simultaneously exhibiting low insertion loss $(\sim 2 \mathrm{~dB})$, which is remarkable since the active region of the device is atomically thin. This modulator performance, among the best reported to date, indicates the enormous potential of graphene for terahertz reconfigurable optoelectronic devices.

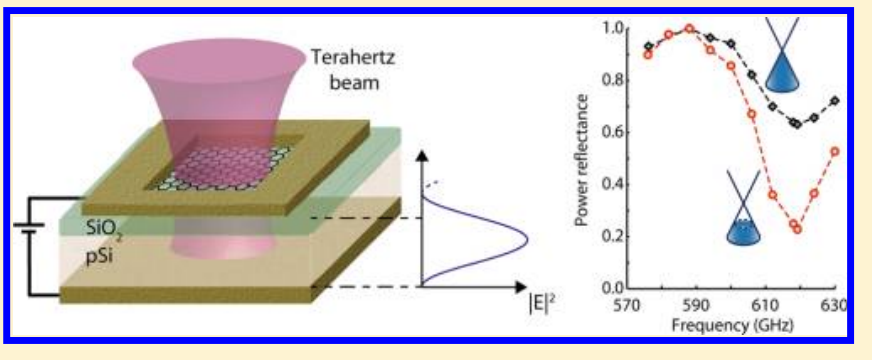

KEYWORDS: Graphene, terahertz, electro-absorption, modulators, filters, active tuning

$\mathrm{T}$ erahertz $(\mathrm{THz})$ technology, owing to its applications in many diverse areas such as astronomy, biomedicine, communications, defense, and so forth, has recently become one of the most dynamic fields of scientific research. ${ }^{1}$ But the limited performance of available terahertz devices restricts the development of many of these applications. Although the past decades have seen increasingly rapid advances in terahertz technologies, particularly emitters and detectors, there is still a dire need for efficient reconfigurable devices such as active filters, modulators, and switches. ${ }^{2}$ Terahertz modulators are structures capable of actively controlling the amplitude (or phase) of the transmitted (or reflected) waves. Applications of these devices include: power stabilization of terahertz sources in spectroscopy, ${ }^{3}$ carrier-wave modulation for terahertz band communication systems, ${ }^{4}$ switches for coded aperture terahertz imaging, ${ }^{5}$ and so forth. Device proposals so far rely on directly modifying the device geometry (e.g., in $\mathrm{MEMS}^{6}$ ) or effectively modifying material properties (primarily optical conductivity) of the tunable elements. For instance, metal-gated semiconductors can provide room-temperature broadband intensity modulation but with low modulation depth ${ }^{7}(\sim 6 \%$ intensity modulation was experimentally demonstrated $\left.{ }^{8}\right)$. By employing graphene as an active "gate", a remarkable broadband modulation of $\sim 20 \%$ was recently reported, ${ }^{9}$ though short of the theoretical expectation of unity modulation due to the quality of the large area graphene currently available. Taking advantage of the plasmonic effects, a modulation depth of $\sim 13 \%$ was also observed at room temperature employing an array of gated graphene stripes; ${ }^{10}$ a higher modulation depth was reported in GaN plasmonic structures but at cryogenic temperatures. ${ }^{11}$ Since larger modulation depths are required for various applications, more efficient structures such as active terahertz metamaterials ${ }^{12}$ are still the most promising alternatives. In many of these metamaterials, the change of conductivity, and thus free carrier absorption in a semiconductor active region, located between periodic metallic patterns (i.e., frequency selective surface), translates into an exceptional control over terahertz wave at frequencies close to the structure intrinsic resonance. This significant tuning can be understood on the basis of an electric field enhancement in the device active region. In this letter, we demonstrate an extremely simple, efficient, and polarization independent terahertz modulator, which employs atomically thin graphene layers as the active material and achieves modulation depth comparable to the state of the art while simultaneously exhibiting much lower losses.

Since its discovery, graphene has attracted much attention in the research community due to its remarkable electrical and mechanical properties. ${ }^{13,14}$ For instance, graphene high-speed transistors with an intrinsic cutoff frequency near $300 \mathrm{GHz}$ were recently demonstrated, ${ }^{15}$ as well as flexible electronics ${ }^{16}$ and so on. In the optical range, graphene has also shown

Received: April 30, 2012

Revised: July 25, 2012

Published: August 3, 2012 
promising device performance, for example, in high-speed infrared (IR) electro-absorption modulators, ${ }^{17}$ transparent electrodes, ${ }^{18,19}$ and photodetectors. ${ }^{20}$ The optical absorption of graphene can be described based on the contributions of inter- and intraband transitions and plasmonic effects, therefore leading to two distinct regions of operation: ${ }^{21}$ first, the IR/ visible range where interband transitions dominate and optical conductivity has either a weak dependence on the Fermi level or is a constant, and second, the lower terahertz range, where optical conductivity is mainly due to intraband transitions, wellrepresented by a Drude model, ${ }^{22}$ and closely follows the electrical conductivity. In the terahertz range, by electrostatically tuning the density-of-states (DOS) available for intraband transitions, terahertz beam transmittance can be effectively controlled. ${ }^{7,9,23}$ Graphene also enables very low insertion loss $(<0.5 \mathrm{~dB})$ owing to its small minimum conductivity. ${ }^{9}$ In addition, taking advantage of plasmonic effects in patterned graphene, the possibility of actively manipulating waves in the upper $\mathrm{THz} /$ far-IR range was recently demonstrated. ${ }^{10}$

The device considered in this work (Figure 1a) consists of a single-layer of graphene on top of $\mathrm{SiO}_{2} / \mathrm{Si}$ substrate with a metal back gate, which also acts as a reflector. The electrical control mechanism is similar to the one previously employed in

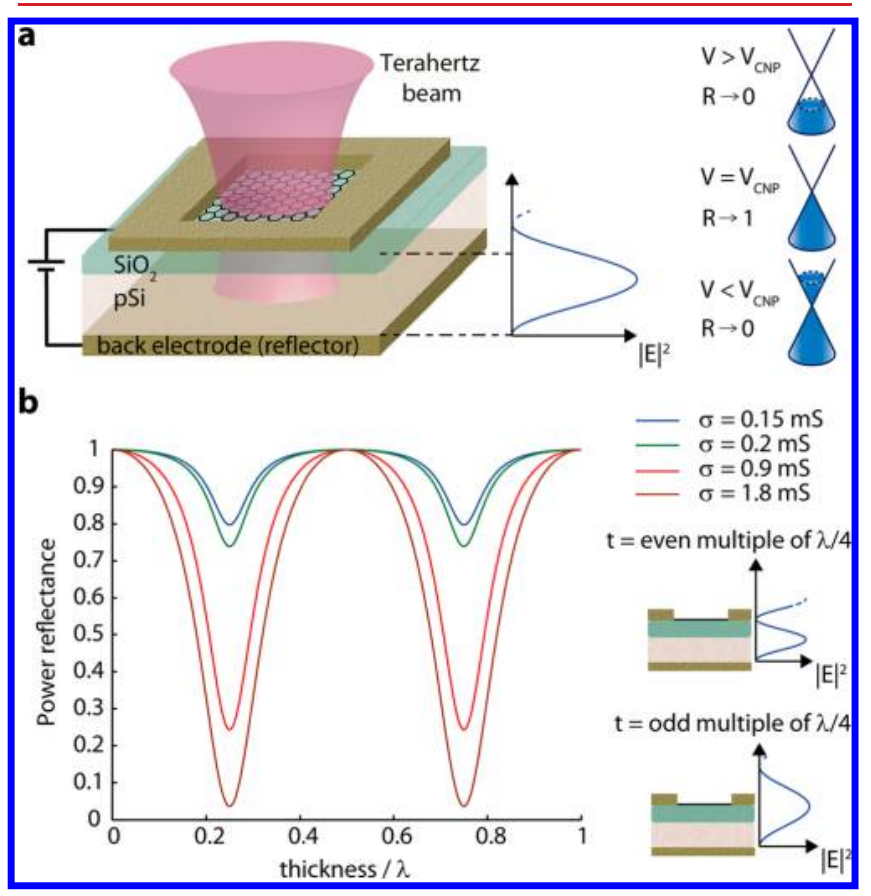

Figure 1. Structure of the graphene electro-absorption modulator and operating principle: (a) Schematic of the terahertz modulator. The single-layer graphene was transferred onto a $\mathrm{SiO}_{2} / p$-Si substrate. The top and bottom metal contacts were employed to tune the graphene conductivity. The electric field intensity exhibits a node at the back contact since it also acts as a reflector. Typical band diagrams for various voltages $(V)$ are depicted on the right. When the Fermi level in graphene is tuned to Dirac point $\left(V=V_{\mathrm{CNP}}\right)$, reflectance is at its maxima. (b) Calculated power reflectance as a function of the substrate optical thickness normalized to terahertz wavelength for different graphene conductivities. When the substrate optical thickness is an even-multiple of a quarter-wavelength, the electric field intensity vanishes in the graphene layer, therefore leading to zero absorption. On the other hand, if the substrate optical thickness is an odd-multiple of a quarter-wavelength, absorption can be extraordinarily tuned. broadband transmittance modulators: by applying a voltage between the top graphene layer and the back metal, the carrier concentration and thus the absorption of terahertz waves in graphene is tuned. Although carriers of the opposite type in graphene are accumulated near the capacitively coupled $\mathrm{SiO}_{2} /$ $\mathrm{Si}$ interface, their influence in modulator performance can be neglected ${ }^{9}$ as the mobility of these carriers in $\mathrm{Si}$ is much smaller than those in graphene. When the Fermi level in graphene is tuned at the Dirac point $\left(V=V_{\mathrm{CNP}}\right)$, the terahertz absorption by the modulator is minimum. On the other hand, when the Fermi level shifts into valence $(\pi)$ or conduction band $\left(\pi^{*}\right)$ of graphene, the density of states available for intraband transitions, and thus the optical absorption, increases. Since the back metal acts as both an electrode and a reflector, the terahertz wave intensity is zero at the back metal; the wave strength in the active graphene layer on top depends on the substrate optical thickness and the terahertz wavelength. As shown in Figure 1b, when the substrate optical thickness is an odd-multiple of the $\mathrm{THz}$ wavelength, the field intensity in graphene is at maxima. Consequently, very large absorption swings, that is, extraordinary modulation, can take place when graphene conductivity is tuned. On the other hand, if the substrate optical thickness is an even-multiple of the $\mathrm{THz}$ wavelength, graphene does not absorb, the modulator reflectance becomes unity and independent of its conductivity, since the field-intensity is zero in the active graphene layer. A similar idea was adopted recently by Lee et al. ${ }^{24}$ to demonstrate a reflectance modulator using graphene in the IR range. However, the observed modulation depth was only $4 \%$ because of the small tunability of graphene absorption in the IR range at normal incidence, which is not the case in the $\mathrm{THz}$ band. ${ }^{7}$ Theoretical analysis of the dependence of reflectance on graphene conductivity and substrate optical thickness is shown in Figure 1b. Given that the optical thickness of the $\mathrm{SiO}_{2}(<100$ $\mathrm{nm})$ is much smaller than the $\mathrm{THz}$ wavelength $(\sim 500 \mu \mathrm{m}$ in air at $600 \mathrm{GHz}$ ), its effect can be neglected. ${ }^{9}$ For the same graphene conductivity swing reported in ref 9 (between 0.2 and $0.9 \mathrm{mS}$ ), a modulation depth of $70 \%$ (4× larger) with an insertion loss of $1.5 \mathrm{~dB}$ is expected. These devices can potentially operate with an insertion loss as low as $1 \mathrm{~dB}$ and a modulation depth approaching $95 \%$ if the large area graphene and insulator quality allow its conductivity to be tuned between the commonly observed DC minimum conductance of $4 e^{2} / h \sim$ $0.15 \mathrm{mS}^{25}$ and a maximum conductivity approaching $1.8 \mathrm{mS}$. Since the top graphene is not patterned, the modulator is intrinsically polarization-independent.

The modulator was fabricated using chemical vapor deposition (CVD) graphene grown on copper. ${ }^{26}$ The $p$-Si substrate is highly resistive $(\rho \sim 1000 \Omega \cdot \mathrm{cm})$ with a $70-\mathrm{nm}$ thick $\mathrm{SiO}_{2}$ grown thermally. Graphene was transferred by means of poly(methyl methacrylate) (PMMA) and wet etch methods. Metal contacts $(\mathrm{Ti} / \mathrm{Au})$ were deposited on graphene and the back side of the Si substrate, as depicted in the device schematic (Figure 1a). Finally, the graphene surface was covered with a thin layer of PMMA. After fabrication, the device was characterized using a terahertz imaging and spectroscopy setup ${ }^{27}$ based on a Virginia Diode, Inc. (VDI) multiplier source, capable of providing continuous wave (CW) radiation in the $570-630 \mathrm{GHz}$ frequency band and a broadband Schottky diode detector. ${ }^{28}$ In all of the experiments the beam was at normal incidence to the samples. A sketch of the setup is presented in Figure 2a. Two control samples were also used: a bare Si substrate and a Si substrate with the metal 


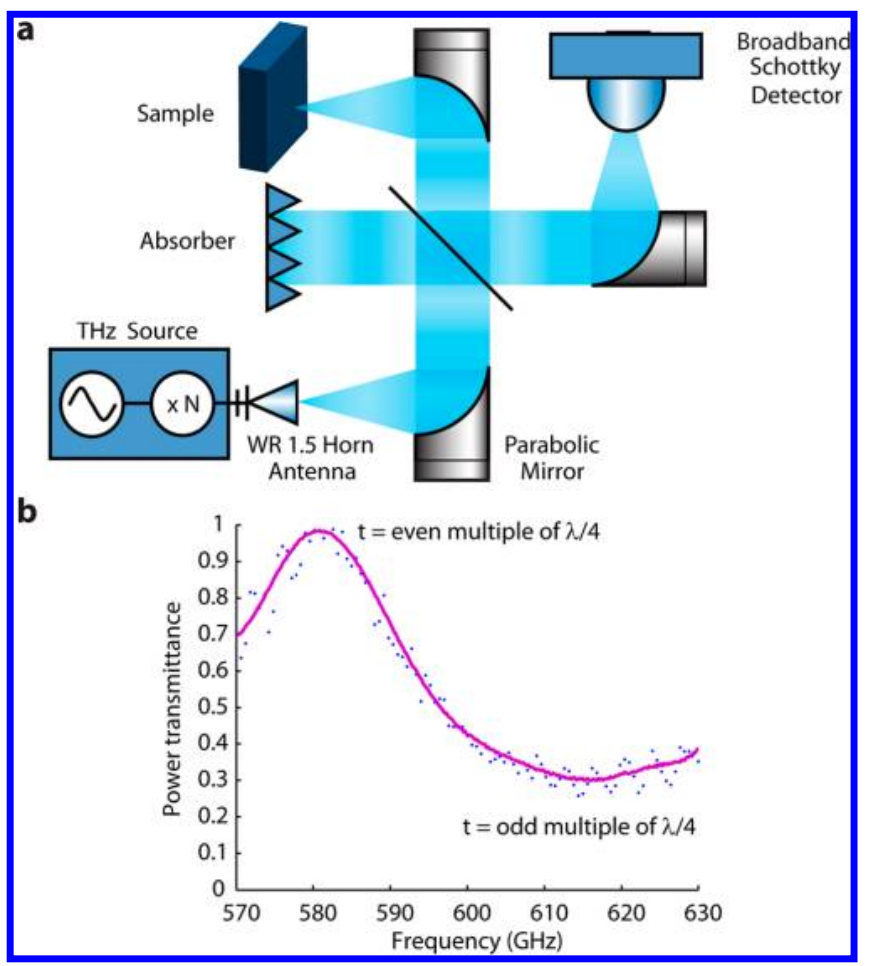

Figure 2. Terahertz setup and transmittance through a bare Si wafer. (a) Schematic of the terahertz imaging and spectroscopy setup. (b) Power transmittance through a bare Si wafer. The frequency at which the substrate optical thickness $(t)$ is an even-multiple of a quarter of the terahertz wavelength is between 580 and $590 \mathrm{GHz}$, while the frequency at which it is an odd-multiple is between 610 and $620 \mathrm{GHz}$.

back reflector. The terahertz transmittance characteristics of the bare $p$-Si wafer are shown in Figure $2 \mathrm{~b}$; the frequencies at which substrate optical thickness is an even (odd) multiple of a quarter-wavelength were found to be $\sim 590(620) \mathrm{GHz}$. The device intensity reflectance $(R)$ was obtained by normalizing the reflected power to that of the control $\mathrm{Si}$ substrate with a back reflector. Since the device area is $\sim 1 \mathrm{~cm}^{2}$, the modulator static characteristics were measured in the region about $1.5 \mathrm{~mm}$ diameter (the terahertz beam size) that shows higher modulation depth. All measurements were carried out at $300 \mathrm{~K}$.

Reflectance as a function of frequency for various voltages between the top contact to the graphene and the back metal is shown in Figure 3a. The measured reflectance closely follows the expected theoretical trend: at $\sim 590 \mathrm{GHz}$ the reflectance is independent of voltage, while at $\sim 620 \mathrm{GHz}$ it can be extraordinarily controlled. At the frequency with the highest modulation, the normalized reflectance (defined as: $R(V)$ / $\left.R\left(V_{\mathrm{CNP}}\right)\right)$ is plotted as a function of voltage (Figure $\left.3 \mathrm{~b}\right)$. The reflectance maximum was obtained at a voltage of $-10 \mathrm{~V}$, corresponding to the Fermi level in graphene being at the Dirac point $\left(V=V_{\mathrm{CNP}}\right)$. When the voltage increases $\left(V>V_{\mathrm{CNP}}\right)$, the hole concentration in graphene ( $p$-type) rises, the reflectance falls as the available density of states for intraband transitions increase. When $V<V_{\mathrm{CNP}}$, the reflectance also drops as the graphene starts to turn into $n$-type. Sketches of the band diagrams for graphene at different bias conditions are provided in Figure 3b. Since the oxide leakage starts to sharply augment around $20 \mathrm{~V}$, the voltages applied in this work are limited to the range of -20 to $20 \mathrm{~V}$.

Modulation depth, defined as: $100 \times\left|R\left(V_{\mathrm{CNP}}\right)-R(V)\right| /$ $R\left(V_{\mathrm{CNP}}\right)$, as a function of frequency is plotted in Figure $3 \mathrm{c}$. The

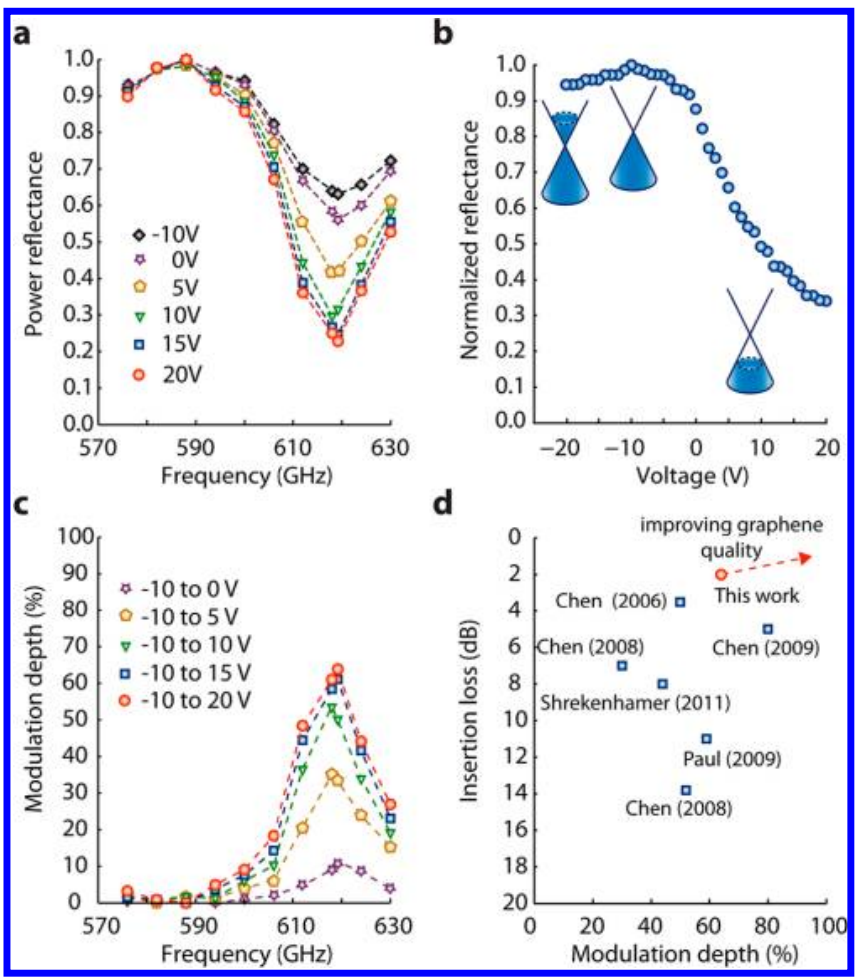

Figure 3. Measured modulator static characteristics. (a) Power reflectance as a function of frequency at different voltages. Around $580-590 \mathrm{GHz}$ the power reflectance is maximum and invariant since, at these frequencies, the substrate optical thickness is an even-multiple of one-quarter of the terahertz wavelength. Near $620 \mathrm{GHz}$ the power reflectance is minimum and voltage tunable because of the tunable absorption in graphene. The insertion loss at $620 \mathrm{GHz}$ is slightly less than $2 \mathrm{~dB}$. (b) Normalized reflectance $\left(R / R\left(V_{\mathrm{CNP}}\right)\right)$ at $620 \mathrm{GHz}$. The Dirac point voltage $V_{\mathrm{CNP}}$ was found to be $-10 \mathrm{~V}$, and band diagrams of graphene are sketched for three bias regimes. (c) Modulation depth as a function of frequency for different voltage swings, the maximum measured modulation depth was $64 \%$. (d) Comparison of modulator performance (modulation depth versus insertion loss) of electrically driven terahertz intensity modulators experimentally demonstrated to date in the literature. ${ }^{12,29-32}$ The graphene-based device presented in this work is extremely simple, and the graphene quality is not optimal; it, nevertheless, delivers one of the best performance considering these two modulator metrics simultaneously.

maximum modulation depth, which occurs near $620 \mathrm{GHz}$, is $64 \%$. The associated insertion loss is slightly $<2 \mathrm{~dB}$ owing to the relatively high minimum conductivity achieved in this particular graphene sample $(\sim 0.3 \mathrm{mS})$. Nevertheless, this is a remarkably low insertion loss, especially considering the structural simplicity of the device and the extraordinary modulation depth achieved. Previously demonstrated experimental results of electrically driven modulators that show similar modulation depths (i.e., modulation depth $>50 \%$ ) exhibit larger insertion loss, as shown in Figure 3d.

The speed of this device is limited by its RC time constant; the modulation amplitude was measured as a function of frequency for voltage swings between $[0,6] \mathrm{V}$ and $[6,12] \mathrm{V}$ (Figure $4 \mathrm{a}$ ). The $3 \mathrm{~dB}$ cutoff frequency was estimated to be $\sim 3.5 \mathrm{kHz}$ and $\sim 4.0 \mathrm{kHz}$, respectively. This trend is expected since the average conductivity in the $[0,6] \mathrm{V}$ range is smaller than that in the $[6,12] \mathrm{V}$ as inferred from Figure 3a. Speed can be improved by reducing the device area to be comparable to the $\mathrm{THz}$ wavelength. 


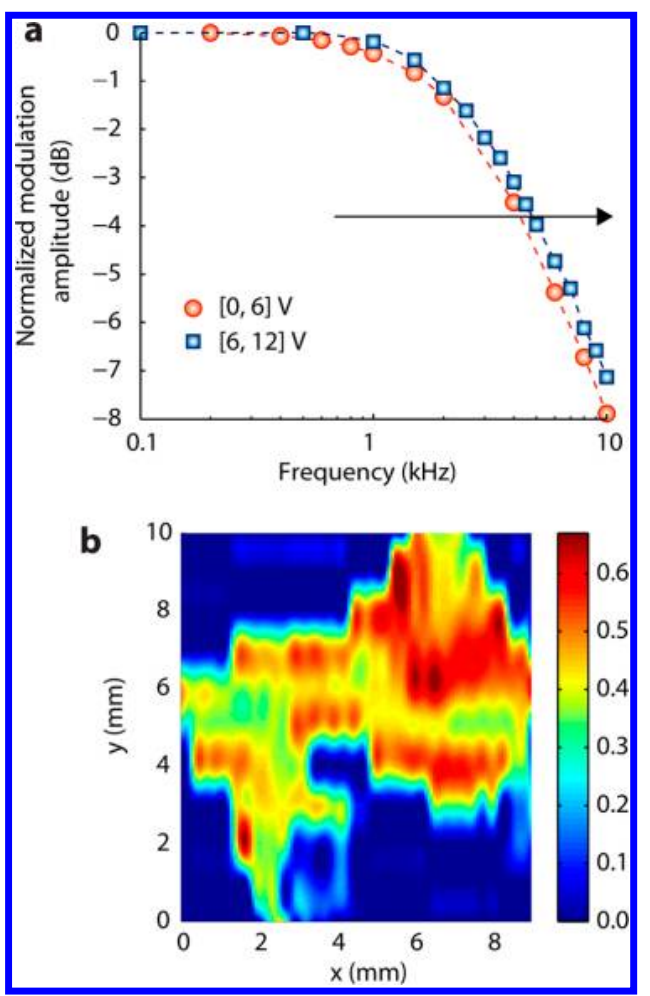

Figure 4. Modulator dynamic characteristics and spatial distribution of modulation depth for a carrier frequency $\sim 620 \mathrm{GHz}$. (a) Normalized modulation amplitude versus switching frequency for two different voltage swings. The voltage swing of $6-12 \mathrm{~V}$ (a higher average graphene conductivity) is further away from $V_{\mathrm{CNP}}$ than that of $0-6 \mathrm{~V}$, which leads to a decrease in the device RC time constant thus a higher $3 \mathrm{~dB}$ cutoff frequency. (b) Spatial distribution of modulation depth; appreciable modulation (ranging from $\sim 40 \%$ to $64 \%$ ) is observed everywhere across the active graphene layer.

Spatial maps of reflectance modulation versus position (Figure 4b), acquired using an $X-Y 2 \mathrm{D}$ mechanical stage, show appreciable modulation (ranging from $\sim 40 \%$ to $64 \%$ ) everywhere across the active graphene layer. The nonuniformity in modulation is attributed to the nonuniform graphene quality (spatially varying minimum conductivity, mobility, thickness, etc.), which most likely stemmed from the fabrication process. $\mathrm{THz}$ imaging in both transmission ${ }^{9}$ and reflection modes can be employed to nondestructively characterize the quality and uniformity of a thin conducting film including graphene, ${ }^{33}$ semiconductors, metals, ${ }^{34}$ and so forth. Benefitting from the field concentration, $\mathrm{THz}$ mapping in the reflection mode offers a better sensitivity.

In conclusion, we have demonstrated exceptional control of terahertz reflectance in a graphene-based electro-absorption modulator, which is also polarization-independent. More than $64 \%$ modulation depth, with a insertion loss $<2 \mathrm{~dB}$ and a modulation speed around $4 \mathrm{kHz}$, is observed, which is so far among the best performances of all experimentally reported electrically driven terahertz intensity modulators. This is remarkable considering that the active region of the device is atomically thin. The ease of fabrication and integration of this intrinsically $2 \mathrm{D}$ material allows the use of graphene as the tunable element in structures like the one presented in this letter or more complex metamaterial structures to further optimize the device performance.

\section{AUTHOR INFORMATION}

\section{Corresponding Author}

*E-mail address: bsensale@nd.edu; hxing@nd.edu

Notes

The authors declare no competing financial interest.

\section{ACKNOWLEDGMENTS}

H.G.X. acknowledges the support from National Science Foundation (CAREER award). D.J. and H.G.X. acknowledge support from the National Science Foundation (ECCS0802125) and from the Office of Naval Research (N0001409-1-0639 and N00014-11-1-0721). L.L. and H.G.X. acknowledge support from the National Science Foundation (ECCS1002088 and ECCS-1202452). M.M.K., L.L., D.J., and H.G.X. also acknowledge the support from the Center of Advanced Diagnostics \& Therapeutics (AD\&T), the Midwest Institute of Nanoelectronics Discovery (MIND), and the Center for Nanoscience and Technology (ND nano) at the University of Notre Dame.

\section{REFERENCES}

(1) Tonouchi, M. Nat. Photon. 2007, 1, 97-105.

(2) Chen, H. T.; O'Hara, J. F.; Azad, A. K.; Taylor, A. J. Laser Photon. Rev. 2011, 5, 513-533.

(3) Choi, B. M. K.; Betterman, A.; Van der Weide, D. W. Philos. Trans. R. Soc. London A 2004, 362, 337-349.

(4) Federici, J.; Moeller, L. J. Appl. Phys. 2010, 107, 111101.

(5) Chan, W. L.; Charan, K.; Takhar, D.; Kelly, K. F.; Baraniuk, R. G.; Mittleman, D. M. Appl. Phys. Lett. 2008, 93, 121105.

(6) Berry, C. W.; Moore, J.; Jarrahi, M. Opt. Express 2011, 19, 1236 1245.

(7) Sensale-Rodriguez, B.; Fang, T.; Yan, R.; Kelly, M. M.; Jena, D.; Liu, L.; Xing, H. G. Appl. Phys. Lett. 2011, 99, 113104.

(8) Kleine-Ostmann, T.; Dawson, P.; Pierz, K.; Hein, G.; Koch, M. Appl. Phys. Lett. 2004, 84, 3555-3557.

(9) Sensale-Rodriguez, B.; Yan, R.; Kelly, M. M.; Fang, T.; Tahy, K.; Hwang, W. S.; Jena, D.; Liu, L.; Xing, H. G. Nat. Commun. 2012, 3, 780.

(10) Ju, L.; Geng, B.; Horng, J.; Girit, C.; Martin, M.; Hao, Z.; Bechtel, H. A.; Liang, X.; Zettl, A.; Shen, Y. R.; Wang, F. Nat. Nanotechnol. 2011, 6, 630-634.

(11) Muravjov, A. V.; Veksler, D. B.; Popov, V. V.; Polischuk, O. V.; Pala, N.; Hu, X.; Gaska, R.; Saxena, H.; Peale, R. E.; Shur, M. S. Appl. Phys. Lett. 2004, 96, 042105.

(12) Chen, H. T.; Padilla, W. J.; Zide, J. M. O.; Gossard, A. C.; Taylor, A. J.; Averitt, R. D. Nature 2006, 444, 597-600.

(13) Geim, A. K.; Novoselov, K. S. Nat. Mater. 2007, 6, 183-191.

(14) Bonaccorso, F.; Sun, Z.; Hasan, T.; Ferrari, A. C. Nat. Photon. 2010, 4, 611-622.

(15) Wu, Y. Q.; Farmer, D. B.; Valdes-Garcia, A.; Zhu, W. J.; Jenkins, K. A.; Dimitrakopoulos, C.; Avouris, P.; Lin, Y. M. IEDM Tech. Dig. 2011, DOI: 10.1109/IEDM.2011.6131601.

(16) Park, J. U.; Nam, S. W.; Lee, M. S.; Lieber, C. M. Nat. Mater. 2012, 11, 120-125.

(17) Liu, M.; Yin, X.; Ulin-Avila, E.; Geng, B.; Zentgraf, T.; Ju, L.; Wang, F.; Zhang, X. Nature 2011, 474, 64-67.

(18) Kim, K. S.; Zhao, Y.; Jang, H.; Lee, S. Y.; Kim, J. M.; Kim, K. S.; Ahn, J. H.; Kim, P.; Choi, J. Y.; Hong, B. H. Nature 2009, 457, 706.

(19) Yan, R.; Zhang, Q.; Li, W.; Calizo, I.; Shen, T.; Richter, C. A.; Hight-Walker, A. R.; Liang, X.; Seabaugh, A.; Jena, D.; Xing, H. G.; Gundlach, D. J.; Nguyen, N. V. Appl. Phys. Lett. 2012, 101, 022105.

(20) Mueller, T.; Xia, F.; Avouris, P. Nat. Photon. 2010, 4, 297-301.

(21) Dawlaty, J. M.; Shivaraman, S.; Chandrashekhar, M.; Rana, F.; Spencer, M. G. Appl. Phys. Lett. 2008, 92, 042116. 
(22) Horng, J.; Chen, C. F.; Geng, B.; Girit, C.; Zhang, Y.; Hao, Z.; Bechtel, H. A.; Martin, M.; Zettl, A.; Crommie, M. F.; Shen, Y. R.; Wang, F. Phys. Rev. B 2011, 83, 165113.

(23) Maeng, I.; Lim, S.; Chae, S. J.; Lee, Y. H.; Choi, H.; Son, J. H. Nano Lett. 2012, 12, 551-555.

(24) Lee, C. C.; Suzuki, S.; Xie, W.; Schibli, T. R. Opt. Express 2012, 20, 5264-5269.

(25) Winnerl, S.; Orlita, M.; Plochocka, P.; Kossacki, P.; Potemski, M.; Winzer, T.; Malic, E.; Knorr, A.; Sprinkle, M.; Berger, C.; de Heer, W. A.; Schneider, H.; Helm, M. Phys. Rev. Lett. 2011, 107, 237401.

(26) Li, X.; Cai, W.; An, J.; Kim, S.; Nah, J.; Yang, D.; Piner, R.; Velamakanni, A.; Jung, I.; Tutuc, E.; Banerjee, S. K.; Colombo, L.; Ruoff, R. S. Science 2009, 324, 1312-1314.

(27) Liu, L.; Hesler, J. L.; Weikle, R. M., II; Wang, T.; Fay, P.; Xing, H. G. Int. J. High Speed Electron. Syst. 2011, 20, 629-638.

(28) Liu, L.; Hesler, J. L.; Xu, H.; Lichtenberger, A. W.; Weikle, R. M., II. IEEE Microwave Wireless Comp. Lett. 2010, 20, 504-506.

(29) Chen, H. T.; Lu, H.; Azad, A.; Averitt, R.; Gossard, A.; Trugman, S.; O’Hara, S.; Taylor, A. Opt. Express 2008, 16, 7641-7648.

(30) Chen, H. T.; Padilla, W. J.; Cich, M. J.; Azad, A. K.; Averitt, R. D.; Taylor, A. J. Nat. Photonics 2009, 3, 148-151.

(31) Paul, O.; Imhof, C.; Lägel, B.; Wolff, S.; Heinrich, J.; Höfling, S.; Forchel, A.; Zengerle, R.; Beigang, R.; Rahm, M. Opt. Express 2009, 17, 819-827.

(32) Shrekenhamer, D.; Rout, S.; Strikwerda, A. C.; Bingham, C.; Averitt, R. D.; Sonkusale, S.; Padilla, W. J. Opt. Express 2011, 19, 9968-9975.

(33) Tomaino, J.; Jameson, A.; Kevek, J.; Paul, M.; van der Zande, A.; Barton, R.; McEuen, P.; Minot, E.; Lee, Y. Opt. Express 2011, 19, 141146.

(34) Henning, P. F.; Homes, C. C.; Maslov, S.; Carr, G. L.; Basov, D. N.; Nikolic, B.; Strongin, M. Phys. Rev. Lett. 1999, 83, 4880-4883. 